\title{
Universiteit
}

Leiden

The Netherlands

\section{On the determination of the structure and tension of the interface between a fluid and a curved hard wall}

Blokhuis, E.M.; Kuipers, J.

\section{Citation}

Blokhuis, E. M., \& Kuipers, J. (2007). On the determination of the structure and tension of the interface between a fluid and a curved hard wall. Journal Of Chemical Physics, 126(5), 054702. doi:10.1063/1.2434161

Version: $\quad$ Not Applicable (or Unknown)

License: $\quad$ Leiden University Non-exclusive license

Downloaded from: https://hdl.handle.net/1887/64309

Note: To cite this publication please use the final published version (if applicable). 


\section{On the determination of the structure and tension of the interface between a fluid and a curved hard wall}

Edgar M. Blokhuis, and Joris Kuipers

Citation: The Journal of Chemical Physics 126, 054702 (2007); doi: 10.1063/1.2434161

View online: https://doi.org/10.1063/1.2434161

View Table of Contents: http://aip.scitation.org/toc/jcp/126/5

Published by the American Institute of Physics

\section{Articles you may be interested in}

The Statistical Mechanical Theory of Surface Tension

The Journal of Chemical Physics 17, 338 (1949); 10.1063/1.1747248

The Effect of Droplet Size on Surface Tension

The Journal of Chemical Physics 17, 333 (1949); 10.1063/1.1747247

Statistical Mechanics of Rigid Spheres

The Journal of Chemical Physics 31, 369 (1959); 10.1063/1.1730361

Surface tension and contact angle of a liquid-solid interface

The Journal of Chemical Physics 74, 2604 (1981); 10.1063/1.441333

An introduction to inhomogeneous liquids, density functional theory, and the wetting transition American Journal of Physics 82, 1119 (2014); 10.1119/1.4890823

Surface thermodynamics of planar, cylindrical, and spherical vapour-liquid interfaces of water The Journal of Chemical Physics 142, 114701 (2015); 10.1063/1.4913371

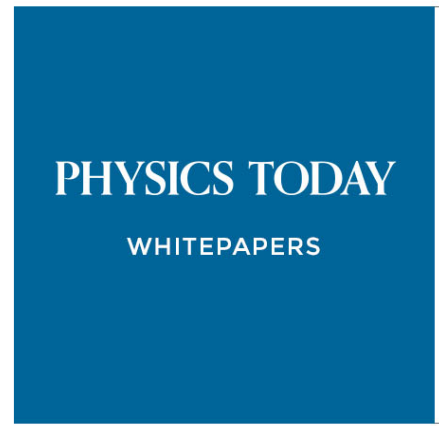

ADVANCED LIGHT CURE ADHESIVES

Take a closer look at what these environmentally friendly adhesive systems can do

\section{READ NOW}

PRESENTED BY Q. MASTERBOND" 


\title{
On the determination of the structure and tension of the interface between a fluid and a curved hard wall
}

\author{
Edgar M. Blokhuis and Joris Kuipers \\ Leiden Institute of Chemistry, P.O. Box 9502, 2300 RA Leiden, The Netherlands
}

(Received 19 October 2006; accepted 21 December 2006; published online 1 February 2007)

\begin{abstract}
The structure and tension of the interface between a fluid and a spherically shaped hard wall are studied theoretically. The authors show the equivalence of different expressions for the surface tension and Tolman length using the squared-gradient model and density functional theory with a nonlocal, integral expression for the interaction between molecules. Even though both these models yield equilibrium density profiles that do not satisfy the wall theorem, they still obey the basic requirement of mechanical equilibrium. The authors trace back the origin of the difference between these two observations to the (lack of) continuity of the cavity function at the hard wall. (C) 2007 American Institute of Physics. [DOI: 10.1063/1.2434161]
\end{abstract}

\section{INTRODUCTION}

For a fluid in contact with a (infinitely) hard wall, the wall theorem (or contact theorem) ${ }^{1}$ states that its density at the wall $\rho^{w}$ is linked to the pressure $p$ as if it were an ideal gas:

$$
p=k_{B} T \rho^{w},
$$

with $T$ the absolute temperature and $k_{B}$ the Boltzmann constant. This equation can be derived by considering the condition of mechanical equilibrium between the solid wall and the fluid phase. It is analogous to the condition of mechanical equilibrium between a coexisting liquid and vapor phase, $p_{\ell}=p_{v}$, with the role of the solid's pressure taken over by $k_{B} T \rho^{w}$. This is particularly evident when we consider a spherically shaped hard wall. Henderson ${ }^{2,3}$ showed that in that case,

$$
-p+k_{B} T \rho^{w}=\frac{2 \sigma(R)}{R}+C(R),
$$

where $R$ is the hard wall radius, $\sigma(R)$ is the radius dependent surface tension, and $C(R) \equiv d \sigma(R) / d R$. One recognizes the right-hand side as the Laplace pressure difference for a liquid droplet in contact with its vapor: ${ }^{4}$

$$
\Delta p=p_{\ell}-p_{v}=\frac{2 \sigma(R)}{R}+C(R) .
$$

As for a liquid droplet, one might expand the radius dependent surface tension in large radii of curvature. To leading order in $1 / R$, one has

$$
\sigma(R)=\sigma\left(1-\frac{2 \delta}{R}+\ldots\right),
$$

where $\sigma$ is the surface tension in the planar limit and where the leading order correction defines the so-called Tolman length $\delta$ introduced by Tolman in $1949 .^{5}$ Inserting the expanded $\sigma(R)$ into Eq. (2) gives

$$
-p+k_{B} T \rho^{w}=\frac{2 \sigma}{R}\left(1-\frac{\delta}{R}+\ldots\right) .
$$

The main purpose of this article are to investigate different expressions for the Tolman length for a fluid near a hard wall and to determine its value using density functional theories of varying degree of sophistication. In particular, we discuss van der Waals squared-gradient theory ${ }^{4}$ and density functional theory (DFT) with a nonlocal, integral expression for the interaction between molecules. ${ }^{6-8}$ Using DFT, Stecki and co-workers ${ }^{9,10}$ showed that for a fluid in contact with a hard wall, the leading order Tolman correction, determined from a plot of $\sigma(R)$ as a function of $1 / R$, is small and positive or negative depending on bulk density and temperature. For the system of a hard sphere fluid near a hard wall, Bryk et $a l .{ }^{11}$ obtained a negative Tolman length, which they showed to be in good agreement with results using "scaled particle theory" (see also Ref. 12).

We are interested in comparing the determination of the Tolman length for the hard wall system with the determination of the Tolman length for a liquid droplet. We show that the Tolman length for a fluid in contact with a hard wall can be determined from the fluid density profile in contact with a planar wall, just as it can in the case of a planar liquid-vapor interface. $^{13-16}$

A second important issue concerns mechanical equilibrium. The Laplace equation for a liquid droplet, Eq. (3), results from a consideration of the mechanical equilibrium between the liquid and vapor phase. An important observation is that for the density functional theories considered, the Laplace equation for a liquid droplet still holds, whereas, in general, they do not obey the wall theorem in Eq. (1) or (2). ${ }^{17}$ This result has also been observed in density functional models for more complex systems in contact with a hard wall. ${ }^{18}$ It is hard to imagine that the condition of mechanical equilibrium may be violated in any reasonable theory. We show that even though the wall theorem need not always be satisfied, the condition of mechanical equilibrium remains 
valid. We further provide explicit expressions for the alternative value of the wall density.

This article is organized as follows: in the next section we consider the thermodynamics necessary to study density functional theories of hard wall systems. We consider quite generally the derivation of the wall theorem for a planar or spherical wall [Eqs. (1) and (2)] and show how the derivation relates to the fundamental requirement of mechanical equilibrium. In Secs. III and IV, we then consider explicitly two density functional theories: squared-gradient theory (Sec. III) and density functional theory that contains an integral expression for the interaction between fluid molecules ${ }^{6-8}$ (Sec. IV). In these two sections we further provide expressions for the surface tension and Tolman length, which are then numerically evaluated. A summary of results is provided in Sec. V.

\section{MECHANICAL EQUILIBRIUM AND THE WALL THEOREM}

There are some similarities but also some important differences when we compare the situation of a fluid in contact with a hard, spherically curved wall with that of a drop of liquid in contact with its vapor. ${ }^{3,19}$

An important distinction between the fluid-wall system and the liquid-vapor system is that one goes from a twophase system to essentially a one-phase system with the wall merely present as a "spectator phase" (not of influence to the thermodynamic state of the fluid phase surrounding it). When investigating a liquid droplet, it is either in a metastable state, the so-called critical nucleus, or in stable equilibrium due to the finite size of the containing vessel. The radius of curvature is then varied either by changing the chemical potential $\mu$ or temperature $T$ (assuming a onecomponent system). For the fluid in contact with a spherical wall, the system is always in equilibrium and the radius of curvature is simply varied as a boundary condition.

We envision the wall as being infinitely repulsive to the fluid beyond some radius $R$; it acts on the fluid through an external potential given by

$$
V_{\text {ext }}(r)= \begin{cases}0 & \text { when } r>R \\ \infty & \text { when } r<R .\end{cases}
$$

This form of the external potential naturally leads us to define the radius $R$ as the radial distance where the molecule's center of mass experiences an infinite repulsion. Some authors ${ }^{11}$ introduce the location of the "actual surface" $R_{\text {act }}$ to account for the fact that the molecule's center of mass is half a diameter away from the surface when it interacts with the hard wall, $R=R_{\text {act }}+d / 2$. Naturally, all physically measurable quantities cannot depend on the definition for the location of the radius $R$, but in this case, it should be chosen such that it reflects the volume available to the liquid's molecules.

To elucidate this matter, let us consider the thermodynamic expression for the free energy (grand potential) of a fluid near a planar hard wall (with area $A$ )

$$
\Omega=-p V_{\ell}+\sigma A
$$

In this expression, $V_{\ell}$ is the volume available to the fluid. If one were to shift the location of the dividing surface, the liquid's volume is altered and the free energy is affected accordingly:

$$
[d \Omega]=-p A[d h],
$$

where $d h$ is the height shift and where the square brackets indicate that we are considering a "notional" shift in the location of the dividing surface which corresponds to a redefinition of the location of the dividing surface without affecting the physical state of the system. ${ }^{4}$ Because the expression for the free energy in Eq. (7) discards the physical properties of the solid phase, we thus find that the free energy is not invariant, $[d \Omega] \neq 0$. This means that we are not free to choose the location of the dividing surface at any position, but should position it such that $V_{\ell}$ corresponds to the actual volume available to the liquid.

Even though the free energy in Eq. (7) is not invariant to a change in the chosen location of the dividing surface, the consideration of a notional shift is useful to derive restrictions on more microscopic models for the free energy. To show this, we consider a notional shift in the location of the radius of a spherically shaped hard wall. The free energy and its notional change are then given by

$$
\begin{aligned}
& \Omega=-p\left(V-\frac{4 \pi}{3} R^{3}\right)+\sigma(R) 4 \pi R^{2}, \\
& {[d \Omega]=\left\{p+\frac{2 \sigma(R)}{R}+C(R)\right\} 4 \pi R^{2}[d R],}
\end{aligned}
$$

where $V$ is the system's total volume. We compare the above result from thermodynamics with a more microscopic model for the free energy. Let us consider, quite generally, the free energy as a functional of the liquid's density $\rho(\mathbf{r})$. It can be written as the sum of a term pertaining to the fluid only $\left(\Omega_{f}\right)$ and a term describing the interaction of the fluid with the wall:

$$
\Omega[\rho]=\Omega_{f}[\rho]+\int d \mathbf{r} \rho(\mathbf{r}) V_{\mathrm{ext}}(\mathbf{r}) .
$$

If we now consider a notional change in the hard wall radius, $[d R]$, the first term in Eq. (10) is unaffected and the only explicit dependence on $R$ stems from the external potential $V_{\text {ext }}(\mathbf{r})=V_{\text {ext }}(r-R)$. For a spherically shaped hard wall, we then have

$$
\begin{aligned}
{[d \Omega] } & =4 \pi \int_{0}^{\infty} d r r^{2} \rho(r) \frac{d V_{\mathrm{ext}}(r)}{d R}[d R] \\
& =-4 \pi R^{2}[d R] \int_{0}^{\infty} d r \rho(r) V_{\mathrm{ext}}^{\prime}(r),
\end{aligned}
$$

where we used $d V_{\text {ext }}(r-R) / d R=-d V_{\text {ext }}(r-R) / d r$ $\equiv-V_{\text {ext }}^{\prime}(r-R)$ and made use of the fact that $V_{\text {ext }}^{\prime}(r)$ is unequal to zero only when $r=R$ for the external potential in Eq. (6). The integration limits may therefore also be replaced by $R^{-}$ 
and $R^{+}$, but we leave them as they are. Comparing Eq. (11) with Eq. (9), one finds

$$
-p-\int_{0}^{\infty} d r \rho(r) V_{\mathrm{ext}}^{\prime}(r)=\frac{2 \sigma(R)}{R}+C(R) .
$$

This equation is an important result. The analogous consideration of the notional change in the radius of a liquid droplet in contact with its vapor leads to the Laplace equation [Eq. (3)]. For a planar surface $(R \rightarrow \infty)$, Eq. (12) reduces to ${ }^{17}$

$$
p=-\int_{-\infty}^{\infty} d z \rho(z) V_{\mathrm{ext}}^{\prime}(z) .
$$

We shall refer to these two equations as the condition of mechanical (or hydrostatic) equilibrium. They can be derived by considering a notional shift of the surface, as we did here, but they can equally well be derived from a consideration of the condition of mechanical equilibrium expressed in terms of the pressure tensor $\mathbf{p}^{4}$

$$
\boldsymbol{\nabla} \cdot \mathbf{p}=-\rho(\mathbf{r}) V_{\mathrm{ext}}^{\prime}(\mathbf{r}) .
$$

For instance, for the planar interface, the above equation reduces to

$$
p_{N}^{\prime}(z)=-\rho(z) V_{\mathrm{ext}}^{\prime}(z),
$$

where $p_{N}(z)$ is the normal component of the pressure tensor which reduces to the uniform pressure $p$ in the bulk. Integrating Eq. (15) over $z$ then derives Eq. (13).

We shall see that the important condition of mechanical equilibrium given by Eq. (12) or (13) is satisfied for the density functional theories that we will discuss in the next sections. One may further note that these expressions for mechanical equilibrium are very closely related to the wall theorem expressed in Eqs. (1) and (2). To show this we rewrite

$$
V_{\mathrm{ext}}^{\prime}(r)=-k_{B} T e^{V_{\mathrm{ext}}(r) / k_{B} T} \frac{d}{d r} e^{-V_{\mathrm{ext}}(r) / k_{B} T} .
$$

The Boltzmann factor for the external potential equals the Heaviside function with its derivative being equal to the Dirac $\delta$ function. Inserting Eq. (16) into Eq. (12), we thus have

$$
-p+k_{B} T \int_{0}^{\infty} d r n(r) \delta(r-R)=\frac{2 \sigma(R)}{R}+C(R),
$$

where we introduced the so-called cavity function (or $y$-function), $n(r)$, defined as the product of the density and the inverse Boltzmann factor for the external potential,

$$
n(r) \equiv \rho(r) e^{V_{\mathrm{ext}}(r) / k_{B} T}
$$

Only if the cavity function is continuous at $r=R$, i.e., $n(R)$ $=n\left(R^{+}\right)=\rho\left(R^{+}\right) \equiv \rho^{w}$, are we able to carry out the integration in Eq. (17) to arrive at Eq. (2):

$$
-p+k_{B} T \rho^{w}=\frac{2 \sigma(R)}{R}+C(R)
$$

The only reason that the validity of Eqs. (12) and (13) in the mean-field density functional theories considered does not necessarily imply the validity of the wall theorem, thus, hinges on whether the cavity function is continuous or not. ${ }^{17}$ The continuity of the cavity function is a fundamental statistical mechanical property, ${ }^{20}$ but it need not necessarily hold in approximate mean-field theories. We shall see that for the density functional theories considered, the cavity function is not continuous and the wall theorem is not satisfied. An exception are those density functional theories in which the so-called weighted density approximation is made. These theories are constructed such that the continuity of the cavity function is implied by the Euler-Lagrange equation. ${ }^{7,9}$

As a side remark, we would like to mention that Lovett and Baus ${ }^{21}$ showed that the wall theorem may also be derived from a virial approach in such a way that nowhere in the proof one needs to rely explicitly on the continuity of the cavity function.

\section{SQUARED-GRADIENT MODEL}

In this section we consider the squared-gradient model for the free energy of a liquid near a hard wall. The free energy as a functional of the density is given by ${ }^{4}$

$$
\Omega[\rho]=\int d \mathbf{r}\left[m|\nabla \rho(\mathbf{r})|^{2}+g(\rho)+\rho(\mathbf{r}) V_{\mathrm{ext}}(\mathbf{r})\right],
$$

where $m$ is the usual coefficient of the squared-gradient term and $g(\rho)$ is the free energy (per unit volume) of a homogeneous fluid with density $\rho$. For explicit calculations, we take $g(\rho)$ to be of the Carnahan-Starling form ${ }^{22}$

$$
\begin{aligned}
g(\rho) & =g_{h s}(\rho)-a \rho^{2} \\
& =k_{B} T \rho \ln (\rho)-\mu \rho+k_{B} T \rho \frac{\left(4 \eta-3 \eta^{2}\right)}{(1-\eta)^{2}}-a \rho^{2},
\end{aligned}
$$

where $\eta \equiv(\pi / 6) \rho d^{3}$ and $a$ is the usual van der Waals parameter to account for the attractive interactions between molecules. The chemical potential $\mu$ determines the bulk fluid density $\rho_{b}$, and it is convenient to use $\rho_{b}$ instead of $\mu$ as the state variable next to $T$. To determine the density profile near a hard wall in squared-gradient theory, we first consider the case of a planar hard wall. The free energy is then

$$
\frac{\Omega[\rho]}{A}=\int_{-\infty}^{\infty} d z\left[m\left[\rho^{\prime}(z)\right]^{2}+g(\rho)+\rho(z) V_{\mathrm{ext}}(z)\right] .
$$

The infinite repulsion of the hard wall is taken into account in two steps. First, we consider the following form for the external potential:

$$
V_{\text {ext }}(z)=\left\{\begin{array}{cc}
0 & \text { when } z>0 \\
k_{B} T V_{0} & \text { when } z<0,
\end{array}\right.
$$

where $V_{0}$ is a very large constant; second, we take the limit $V_{0} \rightarrow \infty$. The Euler-Lagrange equation to minimize the free energy in Eq. (22) reads

$$
2 m \rho^{\prime \prime}(z)=g^{\prime}(\rho)+V_{\text {ext }}(z) .
$$

This differential equation indicates that because $V_{\text {ext }}(z)$ is discontinuous, the resulting density profile exhibits a discontinuous second derivative. However, the profile itself and its first derivative are continuous at $z=0$ : 


$$
\rho\left(0^{-}\right)=\rho\left(0^{+}\right)=\rho^{w}, \quad \rho^{\prime}\left(0^{-}\right)=\rho^{\prime}\left(0^{+}\right) .
$$

Integrating the Euler-Lagrange equation in Eq. (24) gives

$$
m \rho^{\prime}(z)^{2}= \begin{cases}g(\rho)+c_{1} & \text { for } z>0 \\ g(\rho)+k_{B} T V_{0} \rho(z)+c_{2} & \text { for } z<0\end{cases}
$$

The two integration constants $c_{1,2}$ are determined by the behavior of the density profile in the two bulk regions far from the interface. For $z \rightarrow \infty$,

$$
g\left(\rho_{b}\right)=-p \Rightarrow c_{1}=p .
$$

The fluid density in the solid region is exponentially small, $\rho_{s} \simeq \exp \left(-V_{0}\right) \rightarrow 0$. This means that for $z \rightarrow-\infty$,

$$
g\left(\rho_{s}\right) \simeq k_{B} T \rho_{s} \ln \left(\rho_{s}\right) \rightarrow 0 \Rightarrow c_{2}=0 .
$$

Thus we find,

$$
\begin{array}{ll}
m \rho^{\prime}(z)^{2}=g(\rho)+p, & z>0, \\
m \rho^{\prime}(z)^{2}=g(\rho)+k_{B} T V_{0} \rho(z), & z<0 .
\end{array}
$$

Due to the fact that the first derivative is continuous at $z=0$, we have the condition that

$$
g\left(\rho^{w}\right)+p=g\left(\rho^{w}\right)+k_{B} T V_{0} \rho^{w} \Rightarrow \rho^{w}=\frac{p}{k_{B} T V_{0}} \rightarrow 0
$$

The conclusion is therefore

$$
\rho^{w}=0,
$$

which is clearly not in accord with the wall theorem in Eq. (1). In hindsight we might have anticipated this result; it is similar to the condition that the wave function is zero at the boundaries when solving the Schrödinger equation for a particle in a box.

We found that the density profile obtained from the squared-gradient model does not satisfy the wall theorem. One may verify, however, that the squared-gradient model does not violate the condition of mechanical equilibrium, Eq. (13):

$$
p=-\int_{-\infty}^{\infty} d z \rho(z) V_{\mathrm{ext}}^{\prime}(z)=\int_{-\infty}^{\infty} d z \rho(z) k_{B} T V_{0} \delta(z)=k_{B} T V_{0} \rho^{w},
$$

which is indeed the case on account of Eq. (31). That for the squared-gradient model the cavity function $n(z)$ is not continuous can also explicitly be verified from its definition in Eq. (18) and the expression for the density at the wall in Eq. (31):

$$
\begin{aligned}
& n\left(0^{-}\right) \simeq \frac{p}{k_{B} T V_{0}} e^{V_{0}} \rightarrow \infty, \\
& n\left(0^{+}\right) \simeq \frac{p}{k_{B} T V_{0}} \rightarrow 0 .
\end{aligned}
$$

The full fluid density profile can now be determined by taking the limit $V_{0} \rightarrow \infty$. For $z<0$, we have that $\rho(z)=0$, whereas for $z>0$, the density profile is obtained by solving the differential equation in Eq. (29) subject to the boundary condition in Eq. (32). Note that this implies that while for finite $V_{0}$ the first derivative of the density profile is always continuous in $z=0$, in the limit of $V_{0} \rightarrow \infty$, it is not:

$$
\rho^{\prime}\left(0^{-}\right)=0, \quad \rho^{\prime}\left(0^{+}\right)=\sqrt{\frac{p}{m}} .
$$

It is interesting to compare the density profile obtained in the squared-gradient model with the so-called NakanishiFisher model, ${ }^{23}$ which is designed to describe the interaction of a fluid with a wall. Using this model, Nakanishi and Fisher were able to construct a rich wetting phase diagram in terms of two fluid-wall interaction parameters, $h_{1}$ and $g .{ }^{23}$ Besides the presence of (attractive or repulsive) fluid-wall interactions, an important difference with the analysis presented here is that the infinite "hardness" of the wall for $z$ $<0$ is taken into account simply by limiting the integration of the free energy to the region $z>0$. The result is that if we were to set the interaction with the wall to zero $\left(h_{1}=0, g\right.$ $=0$ ), one obtains for the density profile $\rho(z)=\rho_{b}$ everywhere. The Nakanishi-Fisher model is designed specifically to describe the interactions of a fluid with a wall. It is, however, not suited to describe the limit where those interactions vanish.

The applicability of the Nakanishi-Fisher model can also be regarded as a matter of length scales. Suppose we construct a squared-gradient model in which the interaction with the wall is described by an interaction potential with a certain shape and range for $z>0$, and which is strictly infinite for $z<0$. The density profile calculated from such a model could be described in terms of the Nakanishi-Fisher parameters for distances far (compared to the interaction range) from the wall, but nearing the wall, the density profile necessarily approaches zero.

The situation is similar to the theoretical description of a polymer solution in contact with a wall. In the theoretical treatment by de Gennes, ${ }^{24}$ the interaction with the wall is modeled by the "extrapolation length" $(1 / d)$, which is analogous to the parameter $h_{1}$ in the Nakanishi-Fisher model. Setting $1 / d=0$ then again results in a flat monomer density profile. This result is to be contrasted with the analysis by Eisenriegler, who considers a polymer in contact with a purely hard wall, and obtains the boundary condition of vanishing monomer density at the wall. ${ }^{25}$

To determine the radius dependent surface tension and thus Tolman's length, we next consider the spherically shaped hard wall. In spherical coordinates the free energy is given by

$$
\Omega[\rho]=4 \pi \int_{0}^{\infty} d r r^{2}\left[m \rho^{\prime}(r)^{2}+g(\rho)+\rho(r) V_{\mathrm{ext}}(r)\right],
$$

with the Euler-Lagrange equation

$$
2 m \rho^{\prime \prime}(r)=-\frac{4 m}{r} \rho^{\prime}(r)+g^{\prime}(\rho)+V_{\text {ext }}(r) .
$$

The presence of the infinitely hard wall again leads to the boundary condition $\rho(R)=\rho^{w}=0$. The radius dependent surface tension $\sigma(R)$ is calculated by inserting the density profile determined by the above differential equation into the free energy: 


$$
\sigma(R)=\int_{0}^{\infty} d z\left(1+\frac{z}{R}\right)^{2}\left[m\left[\rho^{\prime}(z)\right]^{2}+g(\rho)+p\right]
$$

where we have introduced $z \equiv r-R$ as the (radial) distance to the wall. To determine the Tolman length, we expand $\sigma(R)$ and the density profile in $1 / R$ :

$$
\rho(z)=\rho_{0}(z)+\rho_{1}(z) \frac{1}{R}+\ldots .
$$

The derivation of explicit expressions for $\sigma$ and $\delta \sigma$ in terms of the density profile follows very closely the analogous derivation in Ref. 15 of these coefficients for a spherical liquidvapor surface. The only distinction lies in the fact that the integration over the volume now runs from $z=0$ to $z=\infty$, which is to be expected, and that there is no term associated with $\mu_{1}$, the leading order term in an expansion in $1 / R$ of the chemical potential. The latter is a direct consequence of the fact that the chemical potential in the fluid is constant, independent of the radius $R$.

From the analysis in Ref. 15, we conclude that the surface tension of the planar interface and Tolman length are thus given by

$$
\begin{aligned}
& \sigma=2 m \int_{0}^{\infty} d z\left[\rho_{0}^{\prime}(z)\right]^{2}, \\
& \delta \sigma=-2 m \int_{0}^{\infty} d z z\left[\rho_{0}^{\prime}(z)\right]^{2} .
\end{aligned}
$$

In the next section, these expressions are used to calculate $\sigma$ and $\delta \sigma$.

\section{DENSITY FUNCTIONAL THEORY}

We showed that for the model considered in the previous section, the density at the wall is identically zero. This is a direct consequence of the presence of the squared-gradient term, which gives an infinite contribution to the free energy when the density profile is discontinuous. To allow for such a discontinuity, it therefore seems appropriate to describe the interactions between molecules with the full, non-local integral term: ${ }^{6,7}$

$$
\begin{aligned}
\Omega[\rho]= & \int d \mathbf{r}\left[g_{h s}(\rho)+\rho(\mathbf{r}) V_{\mathrm{ext}}(\mathbf{r})\right] \\
& +\frac{1}{2} \int d \mathbf{r}_{1} \int d \mathbf{r}_{2} U\left(r_{12}\right) \rho\left(\mathbf{r}_{1}\right) \rho\left(\mathbf{r}_{2}\right),
\end{aligned}
$$

where $g_{h s}(\rho)$ is given by the expression in Eq. (21) and $U\left(r_{12}\right)$ is the attractive part of the interaction potential between molecules at a distance $r_{12} \equiv\left|\mathbf{r}_{2}-\mathbf{r}_{1}\right|$. For explicit calculations, we take it to be of the following form: ${ }^{26}$

$$
U\left(r_{12}\right)=\left\{\begin{array}{cc}
0 & \text { when } r_{12}<d \\
-A\left(d / r_{12}\right)^{6} & \text { when } r_{12}>d
\end{array}\right.
$$

to mimic London-dispersion forces. By comparing the free energy in Eq. (20) with Eq. (41), making a gradient expansion in the latter, one may express the parameters $a$ and $m$ in terms of $A$ and $d$ :

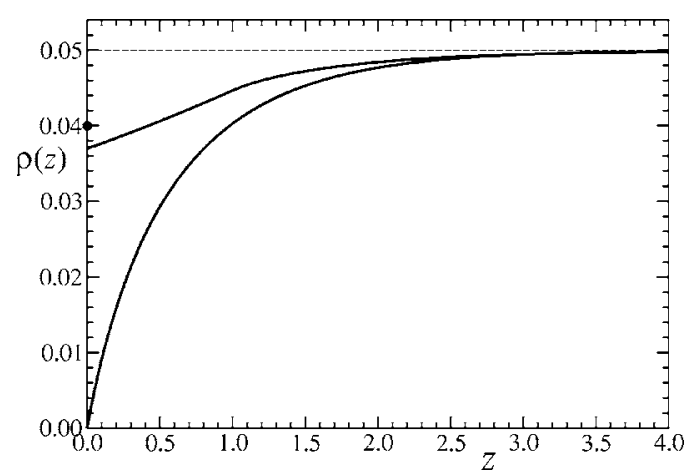

FIG. 1. Density profiles $\rho(z)$ (in units of $1 / d^{3}$ ) as a function of wall distance $z$ (in units of $d$ ) determined by squared-gradient theory (lower solid curve) and density functional theory (upper solid curve). In this example, $T^{*}$ $=0.16$ and $\rho_{b}=0.05$ (dashed line). The solid point indicates the wall density predicted by the wall theorem [Eq. (1)].

$$
\begin{aligned}
& a=-\frac{1}{2} \int d \mathbf{r}_{12} U\left(r_{12}\right)=\frac{2 \pi}{3} A d^{3}, \\
& m=-\frac{1}{12} \int d \mathbf{r}_{12} U\left(r_{12}\right) r_{12}^{2}=\frac{\pi}{3} A d^{5} .
\end{aligned}
$$

It is convenient to express lengths in units of $d$ and energies in units of $a / d^{3}$. The reduced temperature thus becomes $T^{*}$ $\equiv k_{B} T d^{3} / a$.

Again we turn to the planar case first. The free energy is then

$$
\begin{aligned}
\frac{\Omega[\rho]}{A}= & \int_{-\infty}^{\infty} d z\left[g_{h s}(\rho)+\rho(z) V_{\mathrm{ext}}(z)\right] \\
& +\frac{1}{2} \int_{-\infty}^{\infty} d z_{1} \int d \mathbf{r}_{12} U\left(r_{12}\right) \rho\left(z_{1}\right) \rho\left(z_{2}\right),
\end{aligned}
$$

with the Euler-Lagrange equation:

$$
g_{h s}^{\prime}(\rho)+V_{\text {ext }}\left(z_{1}\right)+\int d \mathbf{r}_{12} U\left(r_{12}\right) \rho\left(z_{2}\right)=0 .
$$

For $z<0$, the density profile $\rho(z)=0$, and for $z>0$, one may solve the above integral equation numerically. A typical density profile is shown in Fig. 1. One finds that, in contrast to the squared-gradient model, the density at the wall, $\rho^{w}$, is not equal to zero, but it is also not equal to the value given by the wall theorem in Eq. (1). For the interaction potential in Eq. (42), this observation was already made by van Giessen $e t$ $a l .{ }^{26}$ In Appendix A, we show that the wall density is, instead, determined by

$$
p=-g_{h s}\left(\rho^{w}\right)+\rho^{w} g_{h s}^{\prime}\left(\rho^{w}\right) \equiv p_{h s}\left(\rho^{w}\right) .
$$

This formula was first presented by Parry and Evans, ${ }^{27}$ but there it was derived strictly in the context of the Sullivan model $^{6}$ for the interaction potential between fluid molecules. The analysis in Appendix A shows that this result is independent of the precise form of the interaction potential. The point we like to stress, however, is that, as for the squaredgradient model, the condition of mechanical equilibrium, Eq. (12) or (13), remains satisfied. This is shown explicitly in 
Appendix A. Again, the conclusion is that for this model the cavity function is not continuous at the wall.

To determine the radius dependent surface tension, we now turn to the spherically shaped hard wall. In spherical coordinates the free energy is then

$$
\begin{aligned}
\Omega[\rho]= & 4 \pi \int_{0}^{\infty} d r r^{2}\left[g_{h s}(\rho)+\rho(r) V_{\mathrm{ext}}(r)\right] \\
& +2 \pi \int_{0}^{\infty} d r_{1} r_{1}^{2} \int d \mathbf{r}_{12} U\left(r_{12}\right) \rho\left(r_{1}\right) \rho\left(r_{2}\right),
\end{aligned}
$$

with the Euler-Lagrange equation now given by

$$
g_{h s}^{\prime}(\rho)+V_{\text {ext }}\left(r_{1}\right)+\int d \mathbf{r}_{12} U\left(r_{12}\right) \rho\left(r_{2}\right)=0
$$

The radius dependent surface tension $\sigma(R)$ is derived by inserting the density profile determined by the above differential equation into the free energy:

$$
\begin{aligned}
\sigma(R)= & \int_{0}^{\infty} d z\left(1+\frac{z}{R}\right)^{2}\left[g_{h s}(\rho)-a \rho_{b}^{2}+p\right] \\
& +\frac{1}{2} \int_{0}^{\infty} d z_{1}\left(1+\frac{z_{1}}{R}\right)^{2} \int d \mathbf{r}_{12} U\left(r_{12}\right)\left[\rho\left(r_{1}\right) \rho\left(r_{2}\right)-\rho_{b}^{2}\right] .
\end{aligned}
$$

To obtain explicit expressions for $\sigma$ and $\delta \sigma$, one must again expand $\sigma(R)$ and the density profile in $1 / R$. The derivation follows very closely the analogous derivation in Ref. 28 of these coefficients for a spherical liquid-vapor surface. Following Ref. 28, one finds

$$
\begin{aligned}
& \sigma=-\frac{1}{4} \int_{-\infty}^{\infty} d z_{1} \int d \mathbf{r}_{12} U\left(r_{12}\right) r_{12}^{2}\left(1-s^{2}\right) \rho_{0}^{\prime}\left(z_{1}\right) \rho_{0}^{\prime}\left(z_{2}\right), \\
& \delta \sigma=\frac{1}{4} \int_{-\infty}^{\infty} d z_{1} \int d \mathbf{r}_{12} U\left(r_{12}\right) r_{12}^{2}\left(1-s^{2}\right) z_{1} \rho_{0}^{\prime}\left(z_{1}\right) \rho_{0}^{\prime}\left(z_{2}\right) .
\end{aligned}
$$

The above expression for $\sigma$ is similar to the Triezenberg-Zwanzig ${ }^{29}$ formula for the surface tension.

The integration over $z_{1}$ in Eq. (50) runs over the entire volume, including the region of inhomogeneity. It therefore includes the singular contribution from the derivative of the density profile, $\left.\rho^{\prime}(z)\right|_{\text {sing }}=\rho^{w} \delta(z)$. For numerical evaluation, it is necessary to limit the integration to the liquid region and explicitly take into account the singular contribution. This leads to

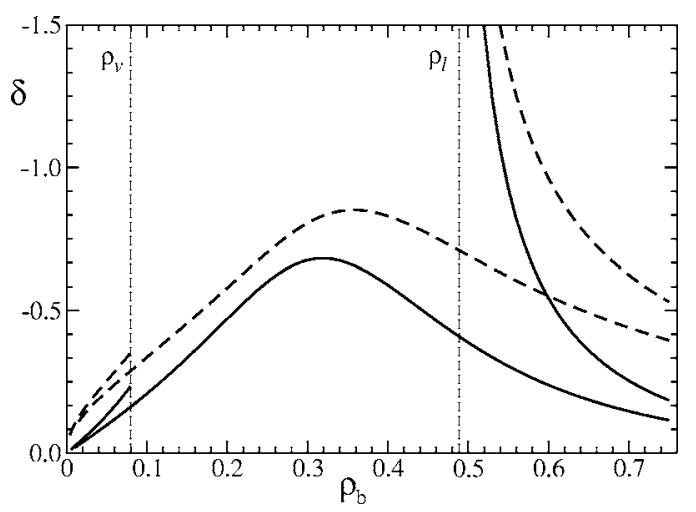

FIG. 2. Tolman length $\delta$ (in units of $d$ ) as a function of bulk density $\rho_{b}$ (in units of $1 / d^{3}$ ) determined by squared-gradient theory (dashed curves) and density functional theory (solid curves) at two temperatures, one below $T_{c}^{*}$ $=0.18015455 \ldots\left(T^{*}=0.16\right)$ and one above $T_{c}\left(T^{*}=0.20\right)$. The curves below $T_{c}$ are those on either side of the vertical dashed lines, which are the limiting bulk densities at coexistence, $\rho_{v}=0.0795594 \ldots$ and $\rho_{\ell}=0.4887348 \ldots$ The curves above $T_{c}$ are those that span the entire density range.

$$
\begin{aligned}
\sigma= & -\frac{1}{4} \int_{0^{+}}^{\infty} d z_{1} \int_{0^{+}} d \mathbf{r}_{12} U\left(r_{12}\right) r_{12}^{2}\left(1-s^{2}\right) \rho_{0}^{\prime}\left(z_{1}\right) \rho_{0}^{\prime}\left(z_{2}\right) \\
& -\frac{\rho^{w}}{2} \int_{0^{+}} d \mathbf{r}_{12} U\left(r_{12}\right) r_{12}^{2}\left(1-s^{2}\right) \rho_{0}^{\prime}\left(s r_{12}\right) \\
& -\frac{\left(\rho^{w}\right)^{2}}{8} \int d \mathbf{r}_{12} U\left(r_{12}\right) r_{12}, \\
\delta \sigma= & \frac{1}{4} \int_{0^{+}}^{\infty} d z_{1} \int_{0^{+}} d \mathbf{r}_{12} U\left(r_{12}\right) r_{12}^{2}\left(1-s^{2}\right) z_{1} \rho_{0}^{\prime}\left(z_{1}\right) \rho_{0}^{\prime}\left(z_{2}\right) \\
& +\frac{\rho^{w}}{4} \int_{0^{+}} d \mathbf{r}_{12} U\left(r_{12}\right) r_{12}^{3} s\left(1-s^{2}\right) \rho_{0}^{\prime}\left(s r_{12}\right),
\end{aligned}
$$

where the lower limit, $0^{+}$, indicates that the integrals are strictly limited to the regions $z_{1}>0$ and $z_{2}>0$. The above expression for $\sigma$, with the singular part explicitly evaluated, is closely related to formula (45) derived by Parry and Evans in Ref. 27.

In Fig. 2 we show, as the solid curves, the Tolman length (in units of $d$ ), numerically determined using the expressions in Eq. (51), as a function of bulk density at two temperatures, one below and one above $T_{c}$. Also drawn in Fig. 2, as the dashed curves, are the results for $\delta$ derived from the squaredgradient model [Eq. (40)] for the same set of parameters. The curves are qualitatively similar to the DFT results and about a factor of 2 larger. Above $T_{c}$ the Tolman length is negative, less than a molecule's diameter, and it exhibits a maximum as a function of the fluid's bulk $T_{c}$ the density range is limited by the densities of the coexisting liquid and vapor phase ( $\rho_{\ell}$ and $\rho_{v}$, vertical dashed lines). On the vapor side, nothing spectacular happens: the Tolman length is negative and less than a molecule's diameter in size. On the liquid side, however, on approaching the coexistence density, the Tolman length diverges.

An interpretation for the divergence of the Tolman length was provided by Evans and co-workers. ${ }^{30-32}$ They showed that a "wetting" layer of vapor is formed between the 
liquid and the hard wall when the coexistence density is approached on the liquid side. It was demonstrated that a crossover radius $R_{c}$ exists such that when $R<R_{c}$, nonanalytic contributions to the surface tension $\sigma(R)$ are present. The crossover radius $R_{c}$ depends critically on the distance to the coexistence density: $R_{c} \equiv 2 \sigma_{\ell v} /(\Delta \rho \Delta \mu)$, with $\sigma_{\ell_{v}}$ the liquidvapor surface tension at coexistence, $\Delta \rho=\rho_{\ell}-\rho_{v}$ the liquidvapor density difference, and $\Delta \mu=\mu-\mu_{\text {coex }}$ the chemical potential distance to liquid-vapor coexistence. The consequence is that while the Tolman length itself remains well defined on approaching the coexistence density, its "usefulness" in the expansion of $\sigma(R)$ in $1 / R$ is restricted to an increasing limited interval, $0<1 / R<1 / R_{c}$.

Furthermore, at coexistence, we have $1 / R_{c}=0$ and the expansion breaks down completely. The leading order correction to the surface tension is not of the Tolman length form $(1 / R)$; the precise expression to replace the Tolman correction is given in Refs. 31 and 32 both for short-ranged forces as well as algebraically decaying interaction forces between fluid molecules. The Tolman length itself is no longer welldefined in the limit $\mu \rightarrow \mu_{\text {coex }}$, which manifests itself in the divergence of $\delta$ as featured in Fig. 2. One may show that the divergence of $\delta$ follows the divergence of the thickness of the intruding wetting layer. This implies that $\delta$ $\propto \ln (\Delta \mu)$ for the short ranged forces of the squared-gradient model $^{31}$ (dashed line), whereas $\delta \propto(\Delta \mu)^{-1 / 3}$, for the dispersion forces of the DFT Ref. 32 (solid line).

It may also be convenient to express $\sigma$ and $\delta \sigma$ in terms of the density profile itself — and not its derivative — to avoid any singular contribution coming from $\rho_{0}^{\prime}(z)$ :

$$
\begin{aligned}
\sigma=\frac{1}{4} \int_{0}^{\infty} d z_{1} \int d \mathbf{r}_{12} U^{\prime}\left(r_{12}\right) r_{12}\left(1-3 s^{2}\right) \rho_{0}\left(z_{1}\right) \rho_{0}\left(z_{2}\right) \\
\delta \sigma=-\frac{1}{8} \int_{0}^{\infty} d z_{1} \int d \mathbf{r}_{12} U^{\prime}\left(r_{12}\right) r_{12}\left(1-3 s^{2}\right) \\
\quad \times\left(z_{1}+z_{2}\right) \rho_{0}\left(z_{1}\right) \rho_{0}\left(z_{2}\right) .
\end{aligned}
$$

As we show in Appendix B these expressions can either be derived from the more general virial expressions for $\sigma$ and $\delta \sigma,{ }^{14,33,34}$ which are valid also beyond the mean-field approximation, or they can be derived by repeated partial integration from Eq. (50). For the results shown in Fig. 2, we have checked that Eqs. (51) and (52) give the same value for $\sigma$ and $\delta \sigma$, within numerical accuracy.

As a further check on our numerical results, we have verified that the Tolman length calculated from Eq. (51) [or Eq. (52)], expressed in terms of the density profile of the planar interface, is equal to the Tolman length obtained directly from the expansion of the radius dependent surface tension, $\sigma(R)=\sigma-2 \delta \sigma / R+\ldots$. In Fig. 3, we show $\sigma(R)$ calculated using Eq. (49) with the density profile determined from numerically solving the differential equation in Eq. (48). The shape of the graph is quite similar to that obtained in Refs. 9 and 12 at low densities. The limiting slope of $\sigma(R)$ near $1 / R=0(-2 \delta \sigma)$ agrees with the value calculated from Eq. (51) (dashed line).

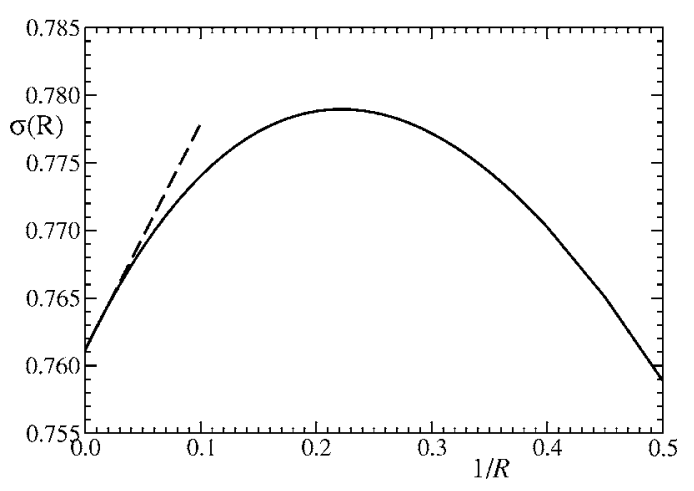

FIG. 3. Radius dependent surface tension $\sigma(R)$ (in units of $10^{-3} a / d^{5}$ ), as a function of the reciprocal radius $1 / R$ of a spherical hard wall, determined by density functional theory. In this example, $T^{*}=0.16$ and $\rho_{b}=0.05$. The dashed line is $\sigma(1-2 \delta / R)$, with $\sigma$ and $\delta$ obtained from the planar density profiles [Eq. (51) or (52)].

\section{SUMMARY}

We have determined density profiles, surface tension, and Tolman length for a fluid in contact with a hard wall using the squared-gradient model and density functional theory with a nonlocal, integral expression for the interaction between molecules. Even though both these models yield equilibrium density profiles which do not satisfy the wall theorem, we showed that they do obey the basic requirement of mechanical equilibrium, thus giving credence to the predictions made.

The expressions for the surface tension and Tolman length are similar to those derived for a liquid-vapor interface, ${ }^{15,28}$ in particular, the Tolman length may again be expressed in terms of the density profile of the planar interface. Furthermore, for the density functional theory, we showed the equivalence between the Triezenberg-Zwanzigtype expression and Kirkwood-Buff-type expression for $\delta$.

Qualitatively, the two models yield similar (numerical) results for the Tolman length as a function of bulk density and temperature: the Tolman length is negative and, generally, less than the molecule's diameter. These results are similar to density functional theory results for the Tolman length of a liquid droplet surrounded by the vapor phase (for a recent review see Ref. 35). An exception is the behavior of the Tolman length on approaching the coexistence density on the liquid side of the phase diagram. Here, the Tolman length diverges, as predicted by Evans and co-workers, ${ }^{30-32}$ due to the formation of a wetting layer of vapor between the liquid and the hard wall.

\section{ACKNOWLEDGMENTS}

The authors would like to express their gratitude to Ben Widom and Jim Henderson for illuminating discussions on the wall theorem.

\section{APPENDIX A: VERIFICATION OF MECHANICAL EQUILIBRIUM}

In this section we derive Eq. (46) and explicitly verify the validity of mechanical equilibrium, Eq. (13), for the den- 
sity functional theory of Sec. IV. Both results are derived from the Euler-Lagrange equation given in Eq. (45):

$$
g_{h s}^{\prime}(\rho)+V_{\text {ext }}\left(z_{1}\right)+\int d \mathbf{r}_{12} U\left(r_{12}\right) \rho\left(z_{2}\right)=0 .
$$

We first derive Eq. (13). This is done by multiplying all the terms in the above Euler-Lagrange equation by $\rho^{\prime}\left(z_{1}\right)$ and subsequently integrating them over $z_{1}$ :

$$
\begin{array}{r}
\int_{-\infty}^{\infty} d z_{1} g_{h s}^{\prime}(\rho) \rho^{\prime}\left(z_{1}\right)+\int_{-\infty}^{\infty} d z_{1} V_{\mathrm{ext}}\left(z_{1}\right) \rho^{\prime}\left(z_{1}\right) \\
+\int_{-\infty}^{\infty} d z_{1} \int d \mathbf{r}_{12} U\left(r_{12}\right) \rho^{\prime}\left(z_{1}\right) \rho\left(z_{2}\right)=0 .
\end{array}
$$

It is important that the integration includes the region of discontinuity at $z=0$, which we have assured by taking the lower limit of the integration at $z_{1}=-\infty$. The first term gives

$$
\int_{-\infty}^{\infty} d z_{1} g_{h s}^{\prime}(\rho) \rho^{\prime}\left(z_{1}\right)=\left[g_{h s}(\rho)\right]_{z_{1}=-\infty}^{z_{1}=\infty}=-p+a \rho_{b}^{2} .
$$

The second term gives

$$
\int_{-\infty}^{\infty} d z_{1} V_{\mathrm{ext}}\left(z_{1}\right) \rho^{\prime}\left(z_{1}\right)=-\int_{-\infty}^{\infty} d z_{1} V_{\mathrm{ext}}^{\prime}\left(z_{1}\right) \rho\left(z_{1}\right) .
$$

The last term gives

$$
\begin{aligned}
\int_{-\infty}^{\infty} d z_{1} \int d \mathbf{r}_{12} U\left(r_{12}\right) \rho^{\prime}\left(z_{1}\right) \rho\left(z_{2}\right) \\
=\left[\int d \mathbf{r}_{12} U\left(r_{12}\right) \rho\left(z_{1}\right) \rho\left(z_{2}\right)\right]_{z_{1}=-\infty}^{z_{1}=\infty} \\
\quad-\int_{-\infty}^{\infty} d z_{1} \int d \mathbf{r}_{12} U\left(r_{12}\right) \rho\left(z_{1}\right) \rho^{\prime}\left(z_{2}\right) \\
=-2 a \rho_{b}^{2}-\int_{-\infty}^{\infty} d z_{1} \int d \mathbf{r}_{12} U\left(r_{12}\right) \rho^{\prime}\left(z_{1}\right) \rho\left(z_{2}\right)=-a \rho_{b}^{2} .
\end{aligned}
$$

We have used $1 \leftrightarrow 2$ symmetry to write the integral expression in the last line as (minus) the same term on the left-hand side. Adding all three terms in Eqs. (A3)-(A5) recovers the condition for mechanical equilibrium, Eq. (13).

Next, we derive Eq. (46). This is achieved by, again, multiplying all the terms in the Euler-Lagrange equation in Eq. (A1) by $\rho^{\prime}\left(z_{1}\right)$, but now the integration over $z_{1}$ is limited to the fluid region:

$$
\int_{0^{+}}^{\infty} d z_{1} g_{h s}^{\prime}(\rho) \rho^{\prime}\left(z_{1}\right)+\int_{0^{+}}^{\infty} d z_{1} \int d \mathbf{r}_{12} U\left(r_{12}\right) \rho^{\prime}\left(z_{1}\right) \rho\left(z_{2}\right)=0 .
$$

The first term now gives

$$
\int_{0^{+}}^{\infty} d z_{1} g_{h s}^{\prime}(\rho) \rho^{\prime}\left(z_{1}\right)=\left[g_{h s}(\rho)\right]_{z_{1}=0^{+}}^{z_{1}=\infty}=-p+a \rho_{b}^{2}-g_{h s}\left(\rho^{w}\right) .
$$

The second term gives

$$
\begin{aligned}
\int_{0^{+}}^{\infty} d z_{1} \int d \mathbf{r}_{12} U\left(r_{12}\right) \rho^{\prime}\left(z_{1}\right) \rho\left(z_{2}\right) \\
=\left[\int d \mathbf{r}_{12} U\left(r_{12}\right) \rho\left(z_{1}\right) \rho\left(z_{2}\right)\right]_{z_{1}=0^{+}}^{z_{1}=\infty} \\
\quad-\int_{0^{+}}^{\infty} d z_{1} \int d \mathbf{r}_{12} U\left(r_{12}\right) \rho\left(z_{1}\right) \rho^{\prime}\left(z_{2}\right) \\
=-2 a \rho_{b}^{2}+\rho^{w} g_{h s}^{\prime}\left(\rho^{w}\right) \\
\quad-\int_{-\infty}^{\infty} d z_{1} \int d \mathbf{r}_{12} U\left(r_{12}\right) \rho\left(z_{1}\right) \rho^{\prime}\left(z_{2}\right),
\end{aligned}
$$

where we have made use of Eq. (A1) with $z_{1}=0^{+}$inserted. A subtle point in this derivation is that the integration over $\mathbf{r}_{12}$ is over the whole space, while the integration over $z_{1}$ is limited to the fluid region and does not include the region of the wall discontinuity. This breaks the $1 \leftrightarrow 2$ symmetry. In order to restore the symmetry, we have therefore extended the integration over $z_{1}$ to the entire volume in the last term. Interchanging $1 \leftrightarrow 2$ in this last term:

$$
\begin{aligned}
& -\int_{-\infty}^{\infty} d z_{1} \int d \mathbf{r}_{12} U\left(r_{12}\right) \rho\left(z_{1}\right) \rho^{\prime}\left(z_{2}\right) \\
& =-\int_{-\infty}^{\infty} d z_{1} \int d \mathbf{r}_{12} U\left(r_{12}\right) \rho^{\prime}\left(z_{1}\right) \rho\left(z_{2}\right) \\
& =\rho^{w} g_{h s}^{\prime}\left(\rho^{w}\right)-\int_{0^{+}}^{\infty} d z_{1} \int d \mathbf{r}_{12} U\left(r_{12}\right) \rho^{\prime}\left(z_{1}\right) \rho\left(z_{2}\right),
\end{aligned}
$$

where we have used the singular contribution to $\left.\rho^{\prime}\left(z_{1}\right)\right|_{\text {sing }}$ $=\rho^{w} \delta\left(z_{1}\right)$, and again used Eq. (A1) for $z_{1}=0^{+}$. Recognizing that the last line contains the same term as the left-hand side in Eq. (A8), with a minus sign, we thus conclude for the second term in Eq. (A6):

$$
\int_{0^{+}}^{\infty} d z_{1} \int d \mathbf{r}_{12} U\left(r_{12}\right) \rho^{\prime}\left(z_{1}\right) \rho\left(z_{2}\right)=-a \rho_{b}^{2}+\rho^{w} g_{h s}^{\prime}\left(\rho^{w}\right) .
$$

This result is added to the result in Eq. (A7) to arrive at Eq. (46):

$$
p=-g_{h s}\left(\rho^{w}\right)+\rho^{w} g_{h s}^{\prime}\left(\rho^{w}\right) .
$$

Note that this derivation does not make any assumption on the precise form of the interaction potential $U\left(r_{12}\right)$ other than that it should be sufficiently short ranged.

\section{APPENDIX B: VIRIAL EXPRESSIONS FOR THE SURFACE TENSION AND TOLMAN LENGTH}

The virial expressions ${ }^{14,33,34}$ for the surface tension and Tolman length of a liquid-vapor interface are given by 


$$
\begin{aligned}
\sigma=\frac{1}{4} \int_{-\infty}^{\infty} d z_{1} \int d \mathbf{r}_{12} U^{\prime}\left(r_{12}\right) r_{12}\left(1-3 s^{2}\right) \rho^{(2)}\left(z_{1}, z_{2}, r_{12}\right) \\
\delta \sigma=-\frac{1}{8} \int_{-\infty}^{\infty} d z_{1} \int d \mathbf{r}_{12} U^{\prime}\left(r_{12}\right) r_{12}\left(1-3 s^{2}\right) \\
\quad \times\left(z_{1}+z_{2}\right) \rho^{(2)}\left(z_{1}, z_{2}, r_{12}\right),
\end{aligned}
$$

where $\rho^{(2)}\left(z_{1}, z_{2}, r_{12}\right)$ is the pair density correlation function for the planar liquid-vapor interface. The first of the above expressions is known as the Kirkwood-Buff formula for the surface tension. ${ }^{33}$ By making the following mean-field approximation for the pair density

$$
\rho^{(2)}\left(z_{1}, z_{2}, r_{12}\right) \approx \rho_{0}\left(z_{1}\right) \rho_{0}\left(z_{2}\right)
$$

we arrive at the expressions for $\sigma$ and $\delta \sigma$ in Eq. (52):

$$
\begin{aligned}
\sigma=\frac{1}{4} \int_{-\infty}^{\infty} d z_{1} \int d \mathbf{r}_{12} U^{\prime}\left(r_{12}\right) r_{12}\left(1-3 s^{2}\right) \rho_{0}\left(z_{1}\right) \rho_{0}\left(z_{2}\right), \\
\delta \sigma=-\frac{1}{8} \int_{-\infty}^{\infty} d z_{1} \int d \mathbf{r}_{12} U^{\prime}\left(r_{12}\right) r_{12}\left(1-3 s^{2}\right) \\
\quad \times\left(2 z_{1}+s r_{12}\right) \rho_{0}\left(z_{1}\right) \rho_{0}\left(z_{2}\right),
\end{aligned}
$$

where it is reminded that $z_{2}=z_{1}+s r_{12}$, with $s=\cos \theta_{12}$. The expressions for $\sigma$ and $\delta \sigma$ in Eqs. (B1) and (B3) are independent of the type of interface; the difference only comes about in the precise form of the pair density [Eq. (B1)] or density profile [Eq. (B3)]. For the case of the wall-fluid interface that we consider here, we have that $\rho_{0}(z)=0$ for $z<0$, so that we might also set the lower limit of the $z_{1}$ integration to $z_{1}=0$. It is, however, convenient to leave the integration over the entire volume.

Our goal is to show the equivalence of the expressions for $\sigma$ and $\delta \sigma$ in Eq. (B3) with those in Eq. (50). We shall limit ourselves to the derivation of the expression for $\delta \sigma$, however. The derivation of the expression for $\sigma$ follows in an analogous way.

As a first step, the expression for $\delta \sigma$ in Eq. (B3) is partially integrated over $r_{12}$. The boundary term vanishes and one finds that

$$
\begin{aligned}
\delta \sigma= & \frac{1}{4} \int_{-\infty}^{\infty} d z_{1} \int d \mathbf{r}_{12} U\left(r_{12}\right)\left(1-3 s^{2}\right)\left(3 z_{1}\right. \\
& \left.+2 s r_{12}\right) \rho_{0}\left(z_{1}\right) \rho_{0}\left(z_{2}\right) \\
& +\frac{1}{8} \int_{-\infty}^{\infty} d z_{1} \int d \mathbf{r}_{12} U\left(r_{12}\right) s r_{12} \\
& \times\left(1-3 s^{2}\right)\left(2 z_{1}+s r_{12}\right) \rho_{0}\left(z_{1}\right) \rho_{0}^{\prime}\left(z_{2}\right) .
\end{aligned}
$$

The integrand in the first term in Eq. (B4) is written as the derivative with respect to $s$ :

$$
\begin{aligned}
\left(1-3 s^{2}\right)\left(3 z_{1}+2 s r_{12}\right)= & \frac{d}{d s}\left[3 z_{1} s\left(1-s^{2}\right)\right. \\
& \left.+\frac{r_{12}}{2}\left(1+2 s^{2}-3 s^{4}\right)\right] .
\end{aligned}
$$

This result is used to partially integrate the first term in Eq. (B4) over $s$. Combining the result with the second term in Eq. (B4) gives

$$
\begin{aligned}
\delta \sigma= & -\frac{1}{8} \int_{-\infty}^{\infty} d z_{1} \int d \mathbf{r}_{12} U\left(r_{12}\right)\left[4 z_{1} s r_{12}\right. \\
& \left.+r_{12}^{2}\left(1+s^{2}\right)\right] \rho_{0}\left(z_{1}\right) \rho_{0}^{\prime}\left(z_{2}\right) .
\end{aligned}
$$

Next, we use $1 \leftrightarrow 2$ symmetry

$$
\begin{aligned}
\delta \sigma= & -\frac{1}{8} \int_{-\infty}^{\infty} d z_{1} \int d \mathbf{r}_{12} U\left(r_{12}\right)\left[-4 z_{1} s r_{12}\right. \\
& \left.+r_{12}^{2}\left(1-3 s^{2}\right)\right] \rho_{0}^{\prime}\left(z_{1}\right) \rho_{0}\left(z_{2}\right)
\end{aligned}
$$

Again, the integrand is written as the derivative with respect to $s$

$$
-4 z_{1} s+r_{12}\left(1-3 s^{2}\right)=\frac{d}{d s}\left[2 z_{1}\left(1-s^{2}\right)+s r_{12}\left(1-s^{2}\right)\right]
$$

which is used to perform yet another partial integration:

$$
\begin{aligned}
\delta \sigma= & \frac{1}{8} \int_{-\infty}^{\infty} d z_{1} \int d \mathbf{r}_{12} U\left(r_{12}\right) r_{12}^{2}\left(1-s^{2}\right) \\
& \times\left(2 z_{1}+s r_{12}\right) \rho_{0}^{\prime}\left(z_{1}\right) \rho_{0}^{\prime}\left(z_{2}\right) .
\end{aligned}
$$

Finally, we note that the $s r_{12}$ term is antisymmetric when we interchange $1 \leftrightarrow 2$, so this term vanishes. We are then left with the result for $\delta \sigma$ anticipated in Eq. (50):

$$
\delta \sigma=\frac{1}{4} \int_{-\infty}^{\infty} d z_{1} \int d \mathbf{r}_{12} U\left(r_{12}\right) r_{12}^{2}\left(1-s^{2}\right) z_{1} \rho_{0}^{\prime}\left(z_{1}\right) \rho_{0}^{\prime}\left(z_{2}\right) .
$$

In connection with the symmetry argument used to derive Eq. (B10) from Eq. (B9), we should mention a subtle point with regard to a similar term present in the expression for $\delta \sigma$ in Eq. (B3). Here, too, one could wonder whether the same argument can be used to show that this term is zero and replace the term $\left(2 z_{1}+s r_{12}\right)$ by $2 z_{1}$. This turns out not to be correct. The reason is that the integral over $z_{1}$ in Eq. (B3) is conditionally convergent: the integrand is zero at $z_{1} \rightarrow \infty$ only when first the integral over $s$ is taken. To make the integral explicitly convergent, it is therefore customary to subtract of the bulk contribution: ${ }^{36}$

$$
\begin{aligned}
\delta \sigma= & -\frac{1}{8} \int_{-\infty}^{\infty} d z_{1} \int d \mathbf{r}_{12} U^{\prime}\left(r_{12}\right) r_{12}\left(1-3 s^{2}\right)\left(2 z_{1}+s r_{12}\right) \\
& \times\left[\rho_{0}\left(z_{1}\right) \rho_{0}\left(z_{2}\right)-\rho_{b}^{2} \Theta\left(z_{1}\right)\right] .
\end{aligned}
$$

The consequence, however, is that the presence of this bulk term breaks the $1 \leftrightarrow 2$ symmetry with the result that the $s r_{12}$ 
term in the above expression is not zero, as an explicit calculation shows:

$$
\begin{aligned}
-\frac{1}{8} \int_{-\infty}^{\infty} & d z_{1} \int d \mathbf{r}_{12} U^{\prime}\left(r_{12}\right) r_{12}\left(1-3 s^{2}\right) s r_{12} \\
& \times\left[\rho_{0}\left(z_{1}\right) \rho_{0}\left(z_{2}\right)-\rho_{b}^{2} \Theta\left(z_{1}\right)\right] \\
1 \leftrightarrow 2 & \frac{1}{8} \int_{-\infty}^{\infty} d z_{1} \int d \mathbf{r}_{12} U^{\prime}\left(r_{12}\right) r_{12}\left(1-3 s^{2}\right) s r_{12} \\
& \times\left[\rho_{0}\left(z_{1}\right) \rho_{0}\left(z_{2}\right)-\rho_{b}^{2} \Theta\left(z_{2}\right)\right] \\
= & \frac{1}{8} \int_{-\infty}^{\infty} d z_{1} \int d \mathbf{r}_{12} U^{\prime}\left(r_{12}\right) r_{12}\left(1-3 s^{2}\right) s r_{12} \\
& \times\left[\rho_{0}\left(z_{1}\right) \rho_{0}\left(z_{2}\right)-\rho_{b}^{2} \Theta\left(z_{1}\right)\right] \\
& +\frac{\rho_{b}^{2}}{8} \int_{-\infty}^{\infty} d z_{1} \int d \mathbf{r}_{12} U^{\prime}\left(r_{12}\right) r_{12}\left(1-3 s^{2}\right) s r_{12} \\
& \times\left[\Theta\left(z_{1}\right)-\Theta\left(z_{2}\right)\right]=\frac{\rho_{b}^{2}}{60} \int d \mathbf{r}_{12} U^{\prime}\left(r_{12}\right) r_{12}^{3} .
\end{aligned}
$$

${ }^{1}$ J. L. Lebowitz, Phys. Fluids 3, 64 (1960).

2 J. R. Henderson, Mol. Phys. 50, 741 (1983).

${ }^{3}$ J. R. Henderson, in Fluid Interfacial Phenomena, edited by C. A. Croxton (Wiley, New York, 1986).

${ }^{4}$ J. S. Rowlinson and B. Widom, Molecular Theory of Capillarity (Clarendon, Oxford, 1982).

${ }^{5}$ R. C. Tolman, J. Chem. Phys. 17, 333 (1949).

${ }^{6}$ D. E. Sullivan, Phys. Rev. B 20, 3991 (1979).

${ }^{7}$ P. Tarazona and R. Evans, Mol. Phys. 52, 847 (1984).

${ }^{8}$ R. Evans, in Liquids at Interfaces, Les Houches Session XLVIII, 1988, edited by J. Charvolin, J. F Joanny, and J. Zinn-Justin (North-Holland, Amsterdam, 1990).

${ }^{9}$ A. Samborski, J. Stecki, and A. Poniewierski, J. Chem. Phys. 98, 8958 (1993).

${ }^{10}$ A. Poniewierski and J. Stecki, J. Chem. Phys. 106, 3358 (1997).

${ }^{11}$ P. Bryk, R. Roth, K. R. Mecke, and S. Dietrich, Phys. Rev. E 68, 031602 (2003).

${ }^{12}$ J. R. Henderson, J. Chem. Phys. 116, 5039 (2002).

${ }^{13}$ M. P. A. Fisher and M. Wortis, Phys. Rev. B 29, 6252 (1984).

${ }^{14}$ E. M. Blokhuis and D. Bedeaux, Physica A 184, 42 (1992).

${ }^{15}$ E. M. Blokhuis and D. Bedeaux, Mol. Phys. 80, 705 (1993).

${ }^{16}$ G. Gompper and S. Zschocke, Europhys. Lett. 16, 731 (1991); , Phys. Rev. A 46, 4386 (1992).

${ }^{17}$ F. van Swol and J. R. Henderson, Phys. Rev. A 40, 2567 (1989).

${ }^{18}$ S. L. Carnie and D. Y. C. Chen, J. Chem. Phys. 74, 1293 (1981); Y. Burak, D. Andelman, and H. Orland, Phys. Rev. E 70, 016102 (2004).

${ }^{19} \mathrm{~J}$. R. Henderson, in Fundamentals of Inhomogeneous Fluids, edited by D. Henderson (Dekker, New York, 1992).

${ }^{20}$ T. Hill, Statistical Mechanics (McGraw-Hill, New York, 1956).

${ }^{21}$ R. Lovett and M. Baus, J. Chem. Phys. 95, 1991 (1991).

${ }^{22}$ N. F. Carnahan and K. E. Starling, Phys. Rev. A 1, 1672 (1970).

${ }^{23}$ H. Nakanishi and M. E. Fisher, Phys. Rev. Lett. 49, 1565 (1982).

${ }^{24}$ P. G. de Gennes, Macromolecules 14, 1637 (1981); 15, 492 (1982).

${ }^{25}$ E. Eisenriegler, Polymers Near Interfaces (World Scientific, Singapore, 1993).

${ }^{26}$ A. E. van Giessen, D. J. Bukman, and B. Widom, J. Colloid Interface Sci. 192, 257 (1997).

${ }^{27}$ A. O. Parry and R. Evans, Mol. Phys. 65, 455 (1988).

${ }^{28}$ A. E. van Giessen, E. M. Blokhuis, and D. J. Bukman, J. Chem. Phys. 108, 1148 (1998).

${ }^{29}$ T. G. Triezenberg and R. Zwanzig, Phys. Rev. Lett. 28, 1183 (1972).

${ }^{30}$ R. Evans, R. Roth, and P. Bryk, Europhys. Lett. 62, 815 (2003).

${ }^{31}$ R. Evans, J. R. Henderson, and R. Roth, J. Chem. Phys. 121, 12074 (2004).

${ }^{32}$ M. C. Stewart and R. Evans, Phys. Rev. E 71, 011602 (2005).

${ }^{33}$ J. G. Kirkwood and F. P. Buff, J. Chem. Phys. 17, 338 (1949).

${ }^{34}$ E. M. Blokhuis and D. Bedeaux, Heterog. Chem. Rev. 1, 55 (1994).

${ }^{35}$ E. M. Blokhuis and J. Kuipers, J. Chem. Phys. 124, 074701 (2006).

${ }^{36}$ E. M. Blokhuis and D. Bedeaux, J. Chem. Phys. 97, 3576 (1992). 